\title{
Local Conformation of the Cellulosic Chain in Solution
}

\author{
Hideyuki YANAI and Takahiro SATO ${ }^{\dagger}$ \\ Department of Macromolecular Science, Osaka University, 1-1 Machikaneyama-cho, Toyonaka 560-0043, Japan
}

(Received August 26, 2005; Accepted October 19, 2005; Published March 15, 2006)

\begin{abstract}
The temperature and molecular weight dependencies of the intrinsic viscosity $[\eta]$ were investigated for cellulose tris(phenyl carbamate) (CTC) in tetrahydrofuran (THF). By the analysis of the $[\eta]$ data in terms of the wormlike cylinder model, the persistence length $q$ of CTC was determined as a function of the temperature. With decreasing the temperature from 25 to $-20^{\circ} \mathrm{C}, q$ increases from 10.5 to $13.7 \mathrm{~nm}$. This temperature dependence of $q$ was successfully explained by the broken wormlike chain model, where each glucose residue in the cellulosic chain is assumed to take left-handed $3 / 1$ or $2 / 1$ helical state and occasionally a kink state generated by a glucosidic bridge angle fluctuation. While the torsional fluctuation in each glucosidic bond is considerably small, there are two energetically favored helical (3/1 and 2/1) states, so that the cellulosic chain may not be regarded as a regular helix in solution. [DOI 10.1295/polymj.38.226]

KEY WORDS Cellulose Derivative / Intrinsic Viscosity / Persistence Length / Broken Wormlike Chain / Local Conformation /
\end{abstract}

Helicity of polymer chains plays important roles in chain rigidity, liquid crystallinity, and chiral discrimination. Recently, several synthetic polymers have been found to take more or less regular helical conformations in solution, and extensive arguments have been made on the relationship among their chemical structure, helicity, chain rigidity, liquid crystallinity, and cholesteric structure. Many review articles deal with recent advances in this subject. ${ }^{1-5}$

Cellulose derivatives are known as semiflexible polymers exhibiting liquid crystallinity ${ }^{6,7}$ and important materials in chiral chromatography. ${ }^{8}$ These features imply helical nature in the cellulosic chain. Conformations of cellulose derivatives in solution were studied both experimentally and theoretically for a long time. However, as mentioned in a previous paper, ${ }^{9}$ their conformational analysis was not an easy task because both chain stiffness and excluded volume effect must be taken into account simultaneously due to their intermediate chain rigidity. Only recent literature provides proper analyses for the conformation of cellulosic chains ${ }^{9,10}$ and also for solution properties (e.g., liquid crystallinity, ${ }^{11}$ viscosity, ${ }^{12}$ and diffusivity ${ }^{13}$ ) based on the properly analyzed conformation.

On the other hand, theoretical studies on the conformation of the cellulosic chain were carried out by Brant et al. ${ }^{14-17}$ in 1970s, who made an extensive conformational energy analysis and found two energetically stable conformations implying left-handed 3/1 and $2 / 1$ helices for the cellulosic chain. However, the characteristic ratio $C_{\infty}$ and its temperature dependence estimated from their conformational energy did not satisfactorily agree with experimental results for cellulose derivatives. ${ }^{15,16}$ The disagreement was argued occasionally afterward, ${ }^{17,18}$ but satisfactory explanation has not been made yet. Thus, the local conformation of the cellulosic chain is still unclear.

In this study, we have revisited this subject in the light of a novel approach recently applied to synthetic helical polymers. ${ }^{5,19,20} \mathrm{We}$ have analyzed the temperature dependence of the persistence length $q$ of a cellulose derivative, cellulose tris(phenyl carbamate) (CTC) in tetrahydrofuran (THF) using the broken wormlike chain model, ${ }^{21}$ and compared the result with the internal rotation potential of Goebel et al. ${ }^{15}$ for the cellulosic chain. For helical polymer chains, monomer units take a same internal rotational state repeatedly along the chain, and the strong conformational correlation among neighboring units is characteristic to this category of polymers. Unfortunately, the above analysis on the temperature dependence of $q$ could not provide information about the degree of the conformational cooperativity for the CTC chain. To supplement the information, we also measured the temperature dependence of the circular dichroism (CD) for CTC in THF which reflects the chiral local conformation, and compare the result with $\mathrm{CD}$ for amylose tris(phenyl carbamate).

\section{EXPERIMENTAL}

\section{CTC Samples}

Four CTC fractions prepared in previous studies ${ }^{9,11}$ were chosen for viscosity measurements. Table I lists the weight-average molecular weight $M_{\mathrm{w}}$ and the ratio of the weight- to number-average molecular weight

${ }^{\dagger}$ To whom correspondence should be addressed (Tel: +81-6-6850-5461, Fax: +81-6-6850-5461, E-mail: tsato@chem.sci.osaka-u.ac.jp). 
Table I. Molecular characteristics of CTC fractions used

\begin{tabular}{|c|c|c|c|c|c|}
\hline Fraction & $M_{\mathrm{w}} / 10^{4}$ & $M_{\mathrm{w}} / M_{\mathrm{n}}^{\mathrm{a}}$ & $\begin{array}{c}\text { Temperature } \\
\left({ }^{\circ} \mathrm{C}\right)\end{array}$ & {$[\eta]^{\mathrm{b}}$} & $k^{\prime}$ \\
\hline \multirow[t]{3}{*}{$\overline{\mathrm{F} 4}$} & 240 & & 25 & 10.47 & 0.41 \\
\hline & & & 0 & 11.93 & 0.37 \\
\hline & & & -20 & 13.43 & 0.39 \\
\hline \multirow[t]{10}{*}{ F6 } & 111 & & 25 & 6.23 & 0.44 \\
\hline & & & 20 & 6.39 & 0.44 \\
\hline & & & 15 & 6.46 & 0.49 \\
\hline & & & 10 & 6.59 & 0.44 \\
\hline & & & 5 & 6.83 & 0.52 \\
\hline & & & 0 & 6.86 & 0.50 \\
\hline & & & -5 & 7.04 & 0.47 \\
\hline & & & -10 & 7.65 & 0.47 \\
\hline & & & -15 & 7.80 & 0.50 \\
\hline & & & -20 & 8.68 & 0.35 \\
\hline \multirow[t]{10}{*}{ F11 } & 33.9 & 1.10 & 25 & 2.22 & 0.35 \\
\hline & & & 20 & 2.26 & 0.39 \\
\hline & & & 15 & 2.39 & 0.33 \\
\hline & & & 10 & 2.45 & 0.33 \\
\hline & & & 5 & 2.50 & 0.35 \\
\hline & & & 0 & 2.50 & 0.37 \\
\hline & & & -5 & 2.63 & 0.34 \\
\hline & & & -10 & 2.70 & 0.35 \\
\hline & & & -15 & 2.76 & 0.35 \\
\hline & & & -20 & 2.80 & 0.38 \\
\hline \multirow[t]{3}{*}{ F18 } & 5.87 & 1.07 & 25 & 0.475 & 0.40 \\
\hline & & & 0 & 0.532 & 0.41 \\
\hline & & & -20 & 0.572 & 0.37 \\
\hline
\end{tabular}

${ }^{\mathrm{a}}$ Estimated by SEC. ${ }^{\mathrm{b}}$ In units of $10^{2} \mathrm{~cm}^{3} / \mathrm{g}$.

$M_{\mathrm{w}} / M_{\mathrm{n}}$ previously determined by static light scattering and SEC, respectively. Values of $M_{\mathrm{w}} / M_{\mathrm{n}}$ for two fractions are equal to or less than 1.1, guaranteeing narrow molecular weight distributions of the fractions used.

\section{Viscometry}

Viscosities of THF solutions of four CTC fractions were measured by using conventional Ubbelohde-type capillary viscometers by changing temperature from -20 to $25^{\circ} \mathrm{C}$. The Huggins and Mead-Fuoss plots were used to determine the intrinsic viscosity $[\eta]$ and the Huggins coefficient $k^{\prime}$ as functions of the temperature and molecular weight.

\section{Static Light Scattering}

Static light scattering measurements were made for fraction $\mathrm{F} 11$ in THF at $20,15,10$, and $5^{\circ} \mathrm{C}$ using a DLS 5000 light scattering apparatus (Otsuka Electronics, Osaka, Japan) equipped with a He-Ne laser. The second virial coefficient $A_{2}$ of this fraction was determined as a function of temperature by using the following equation

$$
\lim _{\theta \rightarrow 0}\left(\frac{K c}{R_{\theta}}\right)=\frac{1}{M_{\mathrm{w}}}+2 A_{2} c+\cdots
$$

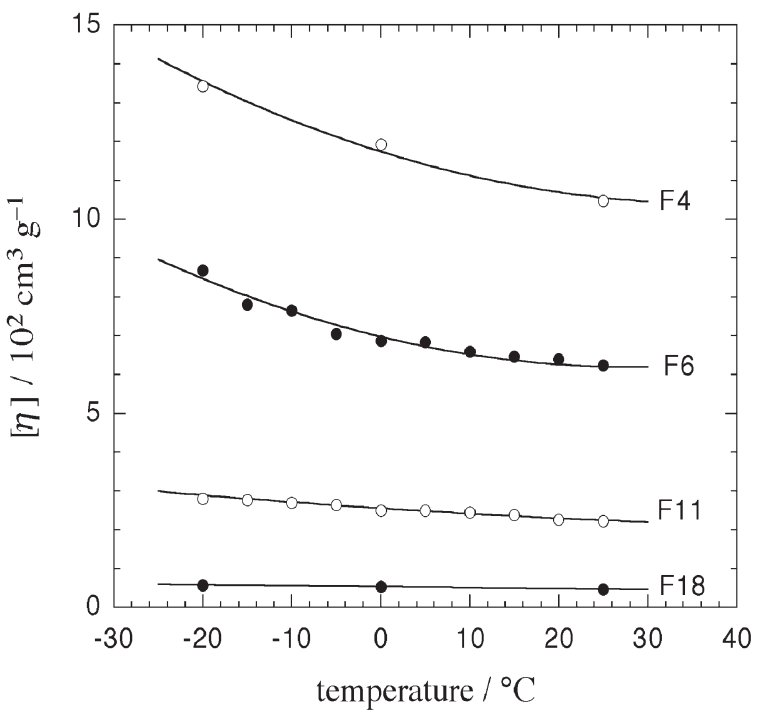

Figure 1. Temperature dependencies of $[\eta]$ for four CTC fractions in THF.

where $K$ is the optical constant, $c$ is the polymer mass concentration, $R_{\theta}$ is the excess Rayleigh ratio at the scattering angle $\theta$. The optical constant $K$ at each temperature was determined so as to give the agreement between $1 / M_{\mathrm{w}}$ of this fraction ( $c f$. Table I) and $K c /$ $R_{\theta}$ at zero $\theta$ and $c$. As shown previously, the linear plot gives a wider linear $c$ region than the root plot, so that the former plot was used to estimate $A_{2}$ as indicated by eq 1 .

\section{Circular Dichroism and UV-vis Absorption Spectros- copy}

Circular dichroism (CD) and UV-vis absorption spectra for dilute THF solutions of a CTC fraction with $M_{\mathrm{w}}=2.2 \times 10^{4}$ were recorded simultaneously on a JASCO J-725 spectropolarimeter equipped with a liquid nitrogen-controlled quartz cell with a path length of $0.5 \mathrm{~cm}$ in a cryostat at wavelength of the incident light between 200 and $600 \mathrm{~nm}$, ranging from 40 to $-100{ }^{\circ} \mathrm{C}$. The polymer concentration was chosen to be $4.4 \times 10^{-5} \mathrm{~g} / \mathrm{cm}^{3}$ (at $25^{\circ} \mathrm{C}$ ).

\section{RESULTS AND DISCUSSION}

\section{Temperature Dependence of the Persistence Length}

Results of $[\eta]$ and $k^{\prime}$ for four CTC fractions in THF at different temperatures are summarized in Table I, and $[\eta]$ data are plotted against temperature in Figure 1. The temperature dependencies of $[\eta]$ are monotonous in THF, which are in a contrast with sigmoidal temperature dependencies of the dielectric relaxation frequency reported for CTC in dioxane. ${ }^{22}$ The chain expansion of CTC with decreasing temperature was reported by several workers. ${ }^{22-26}$ The values of $k^{\prime}$ are normal, indicating no aggregation of CTC in THF 


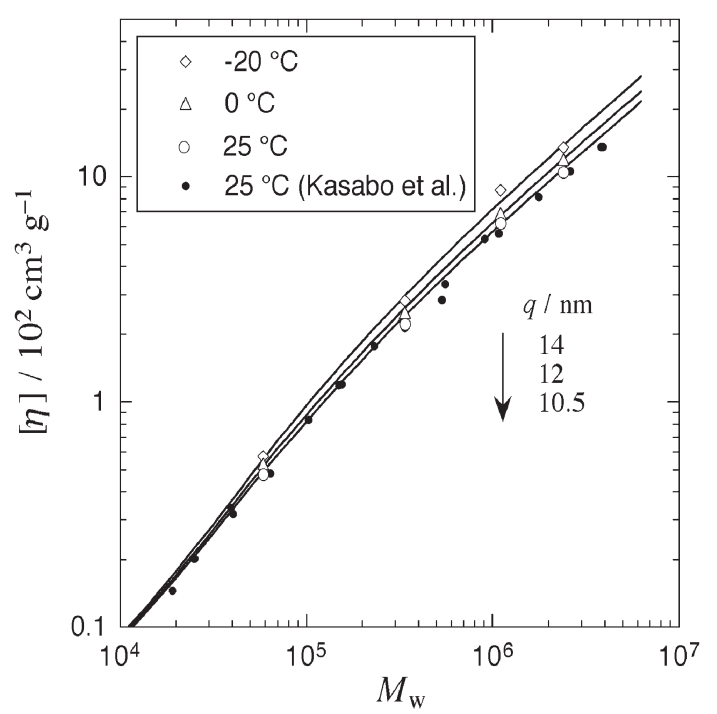

Figure 2. Molecular weight dependencies of $[\eta]$ for CTC in $\mathrm{THF}$ at 25,0 , and $-20^{\circ} \mathrm{C}$; symbols, experimental data (data at $25^{\circ} \mathrm{C}$ being the reproduction of the previous results ${ }^{9}$ ); solid curve, theoretical values calculated by the theory of Yamakawa, Fujii, and Yoshizaki for the wormlike cylinder model ${ }^{27-29}$ combined with the quasi-two-parameter theory for the intramolecular excluded-volume effect using $M_{\mathrm{L}}=1110 \mathrm{~nm}^{-1}, d=2.2 \mathrm{~nm}, B=$ $1.8 \mathrm{~nm}$, and $q$ shown in Figure 4.

upon cooling. Figure 2 plots $[\eta]$ vs. $M_{\mathrm{w}}$ double-logarithmically of CTC in THF at 0 and $-25^{\circ} \mathrm{C}$ as well as the previous results at $25^{\circ} \mathrm{C} .{ }^{9}$ The molecular weight dependencies of $[\eta]$ become slightly stronger with lowering temperature.

Figure 3 plots the second virial coefficient $A_{2}$ for fraction F11 against temperature. As expected for polymer-good solvent systems, $A_{2}$ is almost independent of temperature, which suggests that both inter- and intramolecular excluded-volume effects do not change with temperature.

Previously, ${ }^{9}[\eta]$ data of CTC in THF at $25^{\circ} \mathrm{C}$ were analyzed by using the theory of Yamakawa, Fujii, and Yoshizaki for the wormlike cylinder model ${ }^{27-29}$ combined with the quasi-two-parameter theory ${ }^{29-31}$ for the intramolecular excluded-volume effect to obtain the following wormlike-cylinder parameters: the persistence length $q=10.5 \mathrm{~nm}$, the molar mass per unit contour length $M_{\mathrm{L}}=1110 \mathrm{~nm}^{-1}$, the cylinder diameter $d=2.2 \mathrm{~nm}$, and the strength of the excludedvolume $B=1.8 \mathrm{~nm}$. The $M_{\mathrm{L}}$ value in THF is close to that expected for the energetically stable conformation of the cellulosic chain. Thus we may expect that $M_{\mathrm{L}}$ does not change upon cooling. The $B$ value can be also assumed to be independent of temperature, because $A_{2}$ does not depend on temperature, as shown in Figure 3. Furthermore, $[\eta]$ is insensitive to the value of $d$ unless the axial ratio $L / d$ of the chain is considerably small. Since $L / d$ is larger than 24 at $M_{\mathrm{w}}>$ 58,000 , we may neglect the temperature variation of

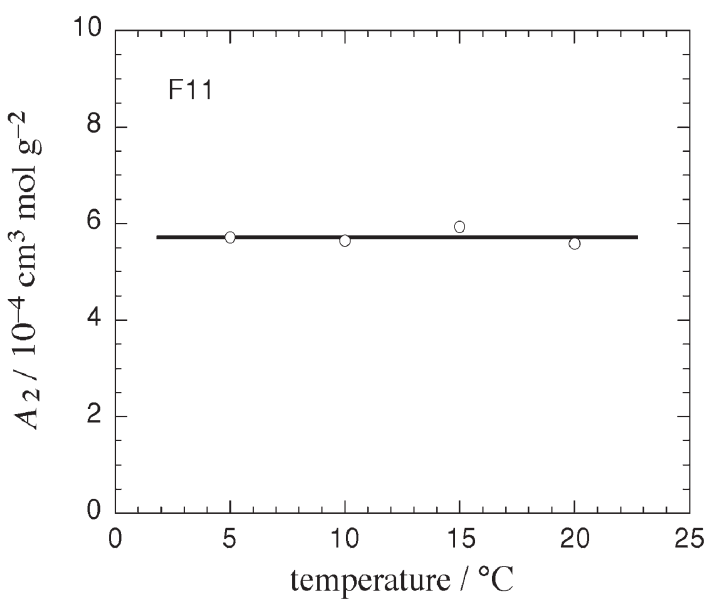

Figure 3. Temperature dependence of $A_{2}$ for fraction F11 in THF.

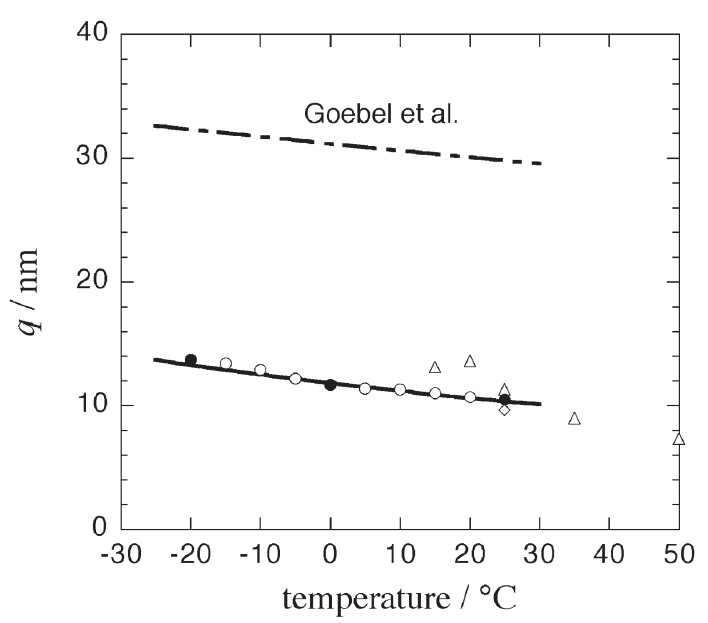

Figure 4. Temperature dependencies of the persistence length for CTC in THF and dioxane; $(\bullet)$, determined from the molecular weight dependence of $[\eta]$ in THF; $(\bigcirc)$, determined from $[\eta]$ data for fractions F6 and F11 in THF; $(\diamond)$, Danhelka et al.'s result ${ }^{10}$ in THF; $(\triangle)$, Gupta et al.'s data ${ }^{22}$ in dioxane; dot-dashed line, theoretical result of $q$ obtained by Goebel et al. $;{ }^{15}$ solid line, the effective persistence length $q$ calculated by eq 4 for the broken wormlike chain model with $\tilde{\theta}_{\mathrm{K}}=40^{\circ}$ and $G^{*}=4.9 \mathrm{~kJ} / \mathrm{mol}$.

$d$. Therefore only $q$ is responsible for the temperature dependence of $[\eta]$. Solid curves shown in Figure 2 indicate the best fit results of the theory where $q$ values were chosen to be 12 and $14 \mathrm{~nm}$ at 0 and $-20^{\circ} \mathrm{C}$, respectively. The $q$ values chosen and the previous result at $25^{\circ} \mathrm{C}$ are shown in Figure 4 by filled circles. In addition, the fit to $[\eta]$ data for fractions F6 and F11 at different temperatures shown in Figure 1 provides us $q$ values shown by unfilled circles in Figure 4 .

There are a number of estimates of $q$ for cellulose derivatives in literature ( $c f$. references cited in ref 9). Figure 4 contains some literature data ${ }^{10,22}$ for CTC in THF and dioxane by a diamond and triangles. Those results are not so much different from ours (filled and 
(a)

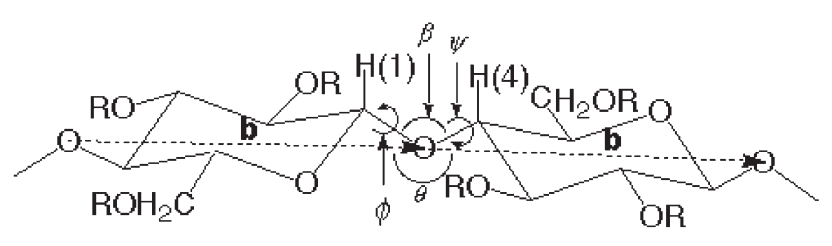

(b)

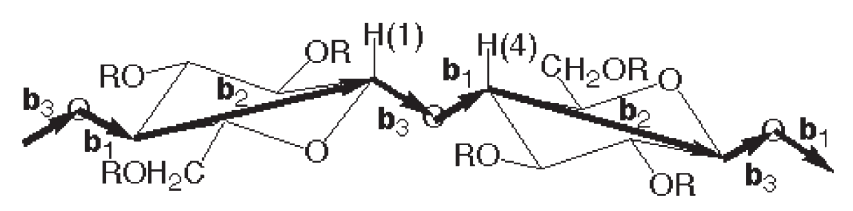

Figure 5. Cellulosic chain represented in terms of (a) the virtual bond $\mathbf{b}$ and (b) three-kind bonds $\mathbf{b}_{1}, \mathbf{b}_{2}$, and $\mathbf{b}_{3}$.

unfilled circles) around room temperatures. Although Gupta et al.'s results $(\triangle)$ look to change abruptly around $25^{\circ} \mathrm{C}$, re-analysis of their dielectric critical frequency data by the moderner theory of Yoshizaki and Yamakawa ${ }^{32}$ yields a temperature dependence of $q$ almost parallel to ours.

\section{Conformation of the CTC Chain}

As depicted in Figure 5a, the cellulosic chain is conventionally regarded to consist of virtual bonds $\mathbf{b}$ indicated by the dotted arrows. From crystallographic data for cellobiose (the dimer model of the cellulosic chain), ${ }^{33}$ the virtual bond length $b$ is estimated to be $0.547 \mathrm{~nm}$. The bond angle $\theta$ and torsional angle $\Phi$ with respect to $\mathbf{b}$ vary with the rotations $\phi$ and $\psi$ about two chemical bonds of the glucosidic bridges $\mathrm{C}(1)-$ $\mathrm{O}(1)$ and $\mathrm{O}(1)-\mathrm{C}\left(4^{\prime}\right)$ indicated in the panel.

Goebel et al. ${ }^{15}$ calculated the internal rotation potential $E(\phi, \psi)$ for cellobiose, considering the repulsive and attractive van der Waals contributions and also electrostatic contribution with assuming that the glucose residues keep the stable $\mathrm{C} 1$ chair-conformation. When the bond angle $\beta$ of the glucosidic bridge (cf. Figure 5a) and the parameter $\Delta$ (characterizing the van der Waals repulsion between interacting two atoms) are chosen to be $116^{\circ}$ (the value for cellobiose in the crystalline state) and $0.02 \mathrm{~nm}$ (a standard value for $\Delta)$, respectively, $E(\phi, \psi)$ takes two energy minima at rotational states which correspond to a lefthanded nearly $3 / 1$ helical $\left(\phi=50^{\circ}, \psi=0^{\circ}\right)$ and a nearly $2 / 1$ helical or the zig-zag $\left(\phi=22^{\circ}, \psi=-35^{\circ}\right)$ states $^{36}$ for the cellulosic chain taking repeatedly each rotational state ( $c f$. Figure 3 in ref 15). The two states however give similar pitches $h$ per the glucose residue, being 0.511 and $0.518 \mathrm{~nm}$, respectively, and the energy barrier between the two states is not so high $(<2 \mathrm{kcal} / \mathrm{mol})$.

The population of the rotational state of the cellulosic chain may be calculated from $E(\phi, \psi)$ according to the Boltzmann law if the internal rotation potential as a function of $\phi$ and $\psi$ is given. Using this population and the geometry of the glucose residue taking the $\mathrm{C} 1$ chair conformation, Goebel et al. ${ }^{15}$ calculated the characteristic ratio $C_{\infty}$ and its temperature coefficient $\mathrm{d} \ln C_{\infty} / \mathrm{d} T$ on the basis of the rotational isomeric state model.

For the wormlike chain model, $C_{\infty}$ is related to the persistence length $q$ by 5,19

$$
q=\left(b^{2} / 2 h\right) C_{\infty}
$$

Figure 4 shows the temperature dependence of $q$ transformed from Goebel et al.'s results of $C_{\infty}$ for $\beta=116^{\circ}$ and $\Delta=0.02 \mathrm{~nm}$, using $b=0.547 \mathrm{~nm}$ and $h=0.514 \mathrm{~nm}$, by the dot-dashed line. The theoretical $q$ values are larger than our and literature experimental results for CTC in THF and dioxane. The disagreement between the theory and experiment for a cellulose derivative ${ }^{23}$ in $C_{\infty}$ has been already mentioned by Goebel et al. themselves. ${ }^{15,16}$

Goebel et al.'s calculation of $C_{\infty}$ is based on the following model; each glucose residue in the cellulosic chain takes two stable rotational states specified by $(\phi, \psi)=\left(50^{\circ}, 0^{\circ}\right)$ (the $3 / 1$ helical state) and $\left(22^{\circ}\right.$, $-35^{\circ}$ ) (the $2 / 1$ helical state) with almost equal probability $\left[E\left(50^{\circ}, 0^{\circ}\right) \approx E\left(22^{\circ},-35^{\circ}\right)\right.$ from Figure 3 in ref 15], and the two $3 / 1$ and $2 / 1$ helical states appear randomly along the chain. If there is some conformational correlation or cooperativeity among neighboring glucose residues, $q$ should be written in the form ${ }^{5,19}$

$$
\frac{1}{q}=\frac{f}{q_{3 / 1}}+\frac{1-f}{q_{2 / 1}}+\frac{1-\cos \tilde{\theta}_{\mathrm{V}}}{\langle l\rangle}
$$

where $q_{3 / 1}$ and $q_{2 / 1}$ are the persistence lengths when all residues in the chain take the $3 / 1$ and $2 / 1$ helical states, respectively, $f$ is the probability that each residue takes the $3 / 1$ state, $\tilde{\theta}_{\mathrm{V}}$ is the angle between the chain-contour axes at the conversion point from the $3 / 1$ to $2 / 1$ sequences, and $\langle l\rangle$ is the arithmetic mean of the average lengths of a $3 / 1$ and $2 / 1$ sequences. The conformational cooperativity increases $\langle l\rangle$ and thus $q$. Therefore, the cooperativity cannot explain the disagreement between experiment and theory in $q$.

Goebel et al. ${ }^{15}$ found that if $\beta$ is slightly increased from $116^{\circ}$ to $125^{\circ}$, new energy minima appear in $E(\phi, \psi)$ in quite different rotational states, i.e., at $(\phi, \psi) \approx\left(0^{\circ}, 180^{\circ}\right)$ or $\left(180^{\circ}, 0^{\circ}\right)(c f$. Figure 4 in 
ref 15). When the cellulosic chain takes these new rotational states, the direction of the virtual bond abruptly changes along the chain. That is, kinks may occasionally appear on the cellulosic chain along with the bond angle fluctuation, as already pointed out by the original authors and the chain may be viewed as a broken wormlike chain. ${ }^{21}$

The effective persistence length for the broken wormlike chain can be written in the form ${ }^{5,19,21}$

$$
\frac{1}{q}=\frac{1}{q_{0}}+\frac{1-\cos \tilde{\theta}_{\mathrm{K}}}{h \exp \left(G^{*} / R T\right)}
$$

where $q_{0}$ is the persistence length for the chain without the kink, $\tilde{\theta}_{\mathrm{K}}$ is the kink angle, $G^{*}$ is the excess free energy (per mole of the repeating unit) when the chain takes the kink conformation, and $R T$ is the gas constant multiplied by the absolute temperature. As mentioned by Mansfield, ${ }^{21}$ the intrinsic viscosity for the normal wormlike and broken wormlike chains are indistinguishable if the parameter $\delta \equiv q\left(1-\cos \tilde{\theta}_{\mathrm{K}}\right)^{2} /$ $2 h \exp \left(G^{*} / R T\right)$ is sufficiently smaller than unity.

The solid line in Figure 4 indicates the theoretical $q$ calculated by eq 4 using $q_{0}$ of Goebel et al. given by the dot-dashed line along with $\tilde{\theta}_{\mathrm{K}}=40^{\circ}$ and $G^{*}=$ $4.9 \mathrm{~kJ} / \mathrm{mol}$, which is favorably compared with the experimental persistence length in THF. The value of $\delta$ is less than 0.08 , so that the broken wormlike chain model is indistinguishable from the wormlike chain model. The kink angle $\tilde{\theta}_{\mathrm{K}}=40^{\circ}$ is realized by introducing in the $3 / 1$ or $2 / 1$ helical sequence a kink conformation $(\phi, \psi) \approx\left(170^{\circ}, 0^{\circ}\right),{ }^{19}$ which is consistent with Goebel et al.'s $E(\phi, \psi)$ at $\beta=125^{\circ}$ (cf. Figure 4 in ref 15). The value of $G^{*}$ indicates that the kink appears in the cellulosic chain every seven glucose residues on average at $25^{\circ} \mathrm{C}$.

\section{Torsional Fluctuation in the CTC Chain}

Recently, Sato et al..$^{5,19}$ estimated the degree of the torsional fluctuation from $q$ data for some synthetic helical polymers. The torsional fluctuation is a basic quantity characterizing the local conformation of polymer chains. Here we estimate the degree of the torsional fluctuation, or the standard deviations of the torsional angles $\phi$ and $\psi$, for the cellulosic chain to compare it with those for other helical polymers.

For this task, the cellulosic chain is regarded to consist of the three kinds of the main-chain bonds $\mathbf{b}_{1}, \mathbf{b}_{2}$, and $\mathbf{b}_{3}$, shown in Figure $5 b$, instead of the virtual bond $\mathbf{b}$ in Panel a. Thus the structure of the cellulosic chain is specified by the three bond lengths $\left(b_{1}, b_{2}\right.$, and $\left.b_{3}\right)$, the three bond angles $\left(\theta_{1}, \theta_{2}\right.$, and $\left.\theta_{3}\right)$, and the three torsional angles $\left(\tilde{\phi}_{1}, \tilde{\phi}_{2}\right.$, and $\left.\tilde{\phi}_{3}\right){ }^{36}$ The virtual bond $\mathbf{b}$ is given by $\mathbf{b}_{1}+\mathbf{b}_{2}+\mathbf{b}_{3}$, while $\beta, \phi$, and $\psi$ correspond to $\theta_{3}, \tilde{\phi}_{3}$, and $\tilde{\phi}_{1}$, respectively. ${ }^{36}$ From crystallographic date for cellobiose, ${ }^{33}$ the bond lengths are given by $b_{1}=0.145 \mathrm{~nm}, b_{2}=0.287 \mathrm{~nm}$, and $b_{3}=0.140 \mathrm{~nm}$, the bond angles by $\theta_{1}=142^{\circ}$, $\theta_{2}=152^{\circ}$, and $\theta_{3}=116^{\circ}$, and the stable torsional angle by $\tilde{\phi}_{1}$ (measured relative to the trans state) $=8.6^{\circ}$ $\tilde{\phi}_{2}=-5.6^{\circ}$, and $\tilde{\phi}_{3}=49^{\circ}$ in the crystalline state. Furthermore, the conformational energy calculation ${ }^{15}$ predicts that the internal rotation potential $E\left(\tilde{\phi}_{1}, \tilde{\phi}_{3}\right)$ takes two minima at $\tilde{\phi}_{1}=9^{\circ}$ and $\tilde{\phi}_{3}=56^{\circ}$ (nearly left-handed $3 / 1$ helix) and at $\tilde{\phi}_{1}=-26^{\circ}$ and $\tilde{\phi}_{3}=$ $29^{\circ}$ (nearly $2 / 1$ helix). ${ }^{36}$

As mentioned above, the (effective) persistence length $q$ of our cellulosic chain model can be calculated by eq 4 where $q_{0}$ is estimated from the right-handed side of eq 3 . Using the above geometrical parameters of the cellulosic chain, we can estimate $\tilde{\theta}_{\mathrm{V}}$ to be $5.8^{\circ},{ }^{19}$ which indicates that the conversion from the $3 / 1$ to $2 / 1$ helical sequences does not essentially change the helix axis of the cellulose chain, and that we can neglect the last term of eq 3 in a good approximation. Therefore, we can calculate $q$ by

$$
\frac{1}{q} \approx \frac{f}{q_{3 / 1}}+\frac{1-f}{q_{2 / 1}}+\frac{1-\cos \tilde{\theta}_{\mathrm{K}}}{h \exp \left(G^{*} / R T\right)}
$$

Here, it is noted that the kink angle $\tilde{\theta}_{\mathrm{K}}$ should not be confused with $\tilde{\theta}_{\mathrm{v}}$.

The persistence length $q_{3 / 1}$ for the $3 / 1$ helix in eq 5 is calculated by

$$
\begin{aligned}
q_{3 / 1} h= & b_{1}^{2}\left(\left[\frac{1}{\mathbf{E}-\mathbf{T}_{1} \mathbf{T}_{2} \mathbf{T}_{3}}\right]_{33}-\frac{1}{2}\right)+b_{2}^{2}\left(\left[\frac{1}{\mathbf{E}-\mathbf{T}_{2} \mathbf{T}_{3} \mathbf{T}_{1}}\right]_{33}-\frac{1}{2}\right)+b_{3}^{2}\left(\left[\frac{1}{\mathbf{E}-\mathbf{T}_{3} \mathbf{T}_{1} \mathbf{T}_{2}}\right]_{33}-\frac{1}{2}\right) \\
& +b_{1} b_{2}\left(\left[\frac{1}{\mathbf{E}-\mathbf{T}_{1} \mathbf{T}_{2} \mathbf{T}_{3}} \mathbf{T}_{1}\right]_{33}+\left[\frac{1}{\mathbf{E}-\mathbf{T}_{2} \mathbf{T}_{3} \mathbf{T}_{1}} \mathbf{T}_{2} \mathbf{T}_{3}\right]_{33}\right) \\
& +b_{2} b_{3}\left(\left[\frac{1}{\mathbf{E}-\mathbf{T}_{2} \mathbf{T}_{3} \mathbf{T}_{1}} \mathbf{T}_{2}\right]_{33}+\left[\frac{1}{\mathbf{E}-\mathbf{T}_{3} \mathbf{T}_{1} \mathbf{T}_{2}} \mathbf{T}_{3} \mathbf{T}_{1}\right]_{33}\right) \\
& +b_{3} b_{1}\left(\left[\frac{1}{\mathbf{E}-\mathbf{T}_{3} \mathbf{T}_{1} \mathbf{T}_{2}} \mathbf{T}_{3}\right]_{33}+\left[\frac{1}{\mathbf{E}-\mathbf{T}_{1} \mathbf{T}_{2} \mathbf{T}_{3}} \mathbf{T}_{1} \mathbf{T}_{2}\right]_{33}\right)
\end{aligned}
$$

where $\mathbf{E}$ is the unit matrix and $\mathbf{T}_{i}(i=1,2$, and 3) are the transformation matrices defined by 
Table II. Standard deviation of the torsional fluctuation for the cellulosic chain and some typical helical polymers (at $25^{\circ} \mathrm{C}$ )

\begin{tabular}{|c|c|}
\hline Polymer & $\left\langle\left(\tilde{\phi}-\tilde{\phi}_{0}\right)^{2}\right\rangle^{1 / 2}$ \\
\hline cellulosic chain & $11.4^{\circ}$ \\
\hline $\operatorname{poly}\{[(R)-3,7 \text {-dimethyloctyl- }(S)-3 \text { methylpentyl }] \text { silylene }\}^{19}$ & $6.2^{\circ}$ \\
\hline $\operatorname{poly}\{[n \text {-hexyl-(S)-3-methylpentyl }] \text { silylene }\}^{19}$ & $17.4^{\circ}$ \\
\hline poly $\left[(4 \text {-carboxyphenyl)acetylene }]^{43}\right.$ & $15^{\circ}$ \\
\hline amylosic chain & $35.4^{\circ}$ \\
\hline
\end{tabular}

$$
\begin{aligned}
& \mathbf{T}_{i}=\left(\begin{array}{ccc}
\left\langle\cos \tilde{\phi}_{i}\right\rangle \cos \theta_{i} & \left\langle\sin \tilde{\phi}_{i}\right\rangle & \left\langle\cos \tilde{\phi}_{i}\right\rangle \sin \theta_{i} \\
\left\langle\sin \tilde{\phi}_{i}\right\rangle \cos \theta_{i} & -\left\langle\cos \tilde{\phi}_{i}\right\rangle & \left\langle\sin \tilde{\phi}_{i}\right\rangle \sin \theta_{i} \\
\sin \theta_{i} & 0 & -\cos \theta_{i}
\end{array}\right) \\
& (i=1,2,3)
\end{aligned}
$$

with $\langle\cdots\rangle$ meaning the thermal average with respect to $\tilde{\phi}_{i}$ calculated using the Boltzmann factor with $E\left(\tilde{\phi}_{1}, \tilde{\phi}_{3}\right)$; the subscript 33 attached to the matrices in eq 6 means the 3,3 element of the matrices. If $E\left(\tilde{\phi}_{1}, \tilde{\phi}_{3}\right)$ is expressed by

$$
E\left(\tilde{\phi}_{1}, \tilde{\phi}_{3}\right)=\frac{1}{2} a_{3 / 1}\left[\left(\tilde{\phi}_{1}-\tilde{\phi}_{1,3 / 1}\right)^{2}+\left(\tilde{\phi}_{3}-\tilde{\phi}_{3,3 / 1}\right)^{2}\right]
$$

in the vicinity of the stable $3 / 1$ helical state, where $\tilde{\phi}_{1,3 / 1}$ and $\tilde{\phi}_{3,3 / 1}$ are the torsional angles in the stable $3 / 1$ helical state and $a_{3 / 1}$ are the force constant, $\left\langle\cos \tilde{\phi}_{i}\right\rangle$ and $\left\langle\sin \tilde{\phi}_{i}\right\rangle$ can be calculated by

$$
\begin{aligned}
& \left\langle\cos \tilde{\phi}_{i}\right\rangle=\cos \tilde{\phi}_{i, 3 / 1} \exp \left(-R T / 2 a_{3 / 1}\right), \\
& \left\langle\sin \tilde{\phi}_{i}\right\rangle=\sin \tilde{\phi}_{i, 3 / 1} \exp \left(-R T / 2 a_{3 / 1}\right) \quad(i=1,3)
\end{aligned}
$$

The rigid glucose ring hinders $\tilde{\phi}_{2}$ from fluctuating. We can calculate $q_{2 / 1}$ in the same way.

Since the two potential wells around the $3 / 1$ and $2 / 1$ helical states are not so much different in the depth and gradient for Goebel et al.'s $E(\phi, \psi)$, we may approximate $a_{3 / 1} \approx a_{2 / 1}$ and $f \approx 1-f \approx 1 / 2$. When we choose $a_{3 / 1}=a_{2 / 1}=9.5 \mathrm{~J} /\left(\mathrm{mol} \cdot \mathrm{deg}^{2}\right), f=$ $1 / 2, \tilde{\theta}_{\mathrm{K}}=40^{\circ}$, and $G^{*}=4.9 \mathrm{~kJ} / \mathrm{mol}$, eq 5 along with eqs 6-8 reproduces the solid line shown in Figure 4. The force constants $a_{3 / 1}$ and $a_{2 / 1}$ chosen yield $11.4^{\circ}$ for the standard deviations of the torsional angles for $\tilde{\phi}_{1}$ and $\tilde{\phi}_{3}$ at $25^{\circ} \mathrm{C}$. As shown in Table II, this degree of the torsional fluctuation for the cellulosic chain is larger than that of a very rigid helical polysilylene derivative, poly $\{[(R)-3,7$-dimethyloctyl- $(S)$ 3-methylpentyl]-silylene $\quad(q=103 \mathrm{~nm})$, but smaller than those of a less rigid helical polysilylene derivative, poly $\{[n$-hexyl-( $S)$-3-methylpentyl $]$-silylene $\}$ $(q=6.1 \mathrm{~nm})$ and a helical polyacetylene derivative poly[(4-carboxyphenyl)acetylene] complexed with an optically active amine $(q=8.6 \mathrm{~nm})$.

As shown in Figures 1 and 2, we observed no sigmoidal temperature nor molecular weight dependencies of $[\eta]$ for CTC in THF, so that we did not consider the conformational cooperativity among neigh- boring repeating units in the CTC chain above. However, this does not necessarily mean that the cellulosic chain taking the $3 / 1$ and $2 / 1$ helical states has no conformational cooperativity. If $q_{3 / 1}$ and $q_{2 / 1}$ are not so much different each other and also $\tilde{\theta}_{\mathrm{V}}$ is small enough, the cooperativity is not reflected on $q$ or $[\eta]$. In fact, $q_{3 / 1} / q_{2 / 1}=0.84$ and $\tilde{\theta}_{\mathrm{V}}=5.8^{\circ}$ for the cellulosic chain from the above estimates, so that the degree of conformational cooperativity may not be estimated from $q$ or $[\eta]$.

Hsu et al. ${ }^{18}$ made a conformational analysis on CTC in a dioxane/methanol mixture ${ }^{25}$ using a different chain model. In their model, each glucose residue can take one of two conformational states referred to as the "stiff" and "flexible" states, and the sequence of the two states along the chain is governed by the Zimm-Bragg theory originally proposed for the helix-coil transition of polypeptides. The "stiff" state was assumed to reside in the existence of cooperative solvophobic and/or hydrogen bonded interactions between the neighboring side-chain phenyl carbamate groups, which restrict the conformational freedom of $\phi$ and $\psi$.

Hsu et al. chose a nearly $3 / 1$ helical state $\left(\phi=70^{\circ}\right.$, $\psi=0^{\circ}$ ) as the most stable conformation for both stiff and flexible states, and $7.0 \mathrm{~J} / \mathrm{mol} \cdot \mathrm{deg}^{2}(=5.5 \mathrm{kcal} /$ $\left.\mathrm{mol} \cdot \mathrm{rad}^{2}\right)$ and $3.8 \mathrm{~J} / \mathrm{mol} \cdot \mathrm{deg}^{2}\left(=3.0 \mathrm{kcal} / \mathrm{mol} \cdot \mathrm{rad}^{2}\right)$ as the force constants for the stiff and flexible states ( $K_{\mathrm{s}}$ and $K_{\mathrm{f}}$ in the original paper), respectively, to fit the molecular weight dependence of the radius of gyration for CTC in a dioxane/methanol mixture. ${ }^{25}$ However, their force constant $K_{\mathrm{f}}$ for the flexible state is too small in comparison with Goebel et al.'s $E(\phi, \psi)$, and cannot be explained by realistic van der Waals radii of atoms. ${ }^{15}$ Furthermore, the force constant $K_{\mathrm{s}}$ for the stiff state also gives a shallower potential well than Goebel et al.'s, although the stiff state should restrict more severely the conformational freedom by the solvophobic and/or hydrogen bonded interactions which was not considered by Goebel et al. To dissolve those inconsistencies, their chain model may need to introduce the kink conformation reducing the chain stiffness, as we did.

Hsu et al. assumed the cooperative "stiff" and "flexible" two state model from a sigmoidal temperature dependence of the dielectric relaxation frequency 
in dioxane $\mathrm{e}^{22}$ and also an unusual molecular weight dependence of the radius of gyration in dioxane/methanol mixture ${ }^{25}$ for CTC. As mentioned above, there are no such peculiar dependencies for CTC in THF that we do not have to introduce the cooperative two state model, at least for CTC in THF. It is interesting to study the different conformational behavior of CTC in THF and dioxane, but we do not discuss this problem here.

\section{Comparison between the Cellulosic and Amylosic Chain Conformations}

Goebel et $a l .{ }^{34}$ calculated also the internal potential $E\left(\tilde{\phi}_{1}, \tilde{\phi}_{3}\right)$ for the amylosic chain. The result has a single shallow minimum at $\left(\tilde{\phi}_{1}, \tilde{\phi}_{3}\right)=\left(-19^{\circ},-44^{\circ}\right)$ corresponding to a left-handed $5 / 1$ helix, which forms a contrast to a rather deep double-well type potential for the cellulosic, in spite of the resemblance in the chemical structure between the two polysaccharides. Brant and Dimpf ${ }^{37}$ further estimated the characteristic ratio $C_{\infty}$ of amylose to be 5.5 from $E\left(\tilde{\phi}_{1}, \tilde{\phi}_{3}\right)$, and the result was favorably compared with experimental data. ${ }^{35}$ The standard deviation of the torsional fluctuation for $\tilde{\phi}_{1}$ and $\tilde{\phi}_{3}$ for amylose can be calculated from this $C_{\infty}$ in a similar way to cellulose. The geometrical parameters of the amylosic chain can be extracted from crystallographic data for methyl $\beta$-Dmaltopyranoside. ${ }^{38}$ As shown in Table II, the result is much larger than that of cellulose due to the shallowness of $E\left(\tilde{\phi}_{1}, \tilde{\phi}_{3}\right) .{ }^{39}$ Although the helical nature of the amylosic chain was pointed out by several authors, ${ }^{17,35,40,41}$ the global conformation of amylose may be regarded as the random coil due to this large torsional fluctuation.

A long time ago, Bitteger and Keilich ${ }^{42}$ observed a strong exciton splitting in circular dichroism (CD) of phenylcarbamate-substituted amylose and maltose (the dimer of amylose) near $230 \mathrm{~nm}$ but a weak simple negative Cotton effect on $\mathrm{CD}$ of phenylcarbamate-substituted cellulose and cellobiose at a similar wavelength. From these observations, they proposed a locally helical conformation for the amylosic chain and a nearly planar conformation for the cellulosic chain.

Figure 6 shows CD and UV spectra of a CTC fraction with $M_{\mathrm{w}}=2.2 \times 10^{4}$ in THF at temperatures from 40 to $-100^{\circ} \mathrm{C}$. Above $-20^{\circ} \mathrm{C}$, the $\mathrm{CD}$ spectrum exhibits a simple negative Cotton effect as reported by Bitteger and Keilich. On the other hand, below $-40{ }^{\circ} \mathrm{C}$ the CD spectra of CTC shows a exciton splitting similar to that for amylose tris(phenyl carbamate) (ATC) in dioxane at room temperature. ${ }^{42}$ The exciton splitting in $\mathrm{CD}$ indicates a helical arrangement of phenyl carbamate chromophores attaching to the cellulosic and amylosic chains, and the helical arrangement probably arises from a locally helical conforma-

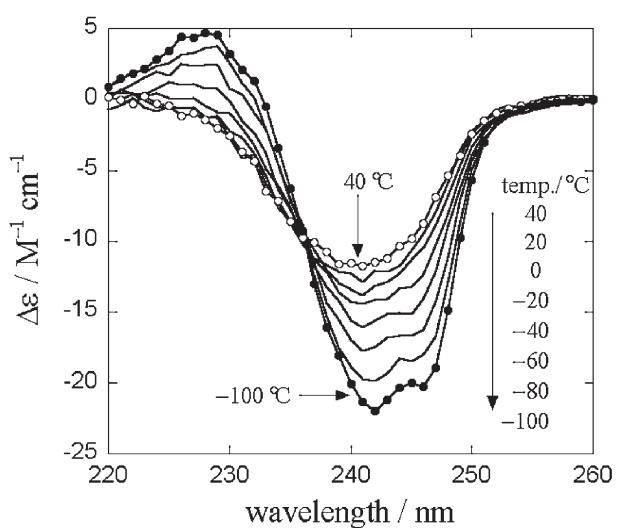

(a)

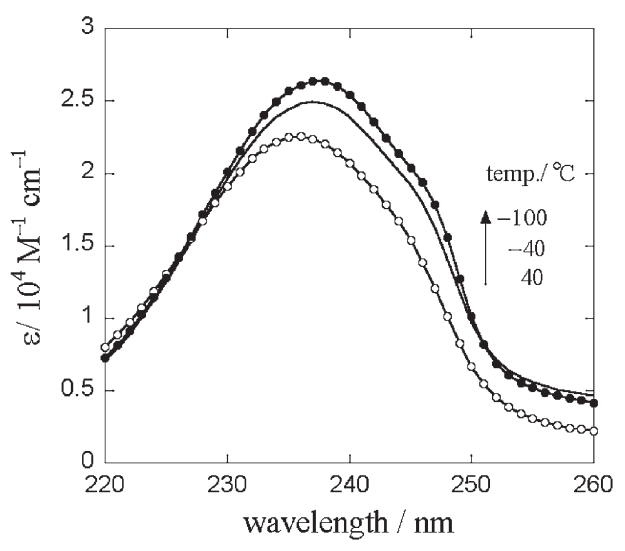

(b)

Figure 6. $\mathrm{CD}$ and UV-vis absorption spectra of a CTC sample with $M_{\mathrm{w}}=2.2 \times 10^{4}$ in THF $\left(c=4.4 \times 10^{-5} \mathrm{~g} / \mathrm{cm}^{3}\right.$ at $25^{\circ} \mathrm{C}$ ) ranging from 40 to $-100{ }^{\circ} \mathrm{C}$, where $\varepsilon$ is the (monomer-unit) molar extinction coefficient, and $\Delta \varepsilon$ is the difference in $\varepsilon$ between right- and left-handed circularly polarized light.

tion of both main chains. It is noted that Bitteger and Keilich $^{42}$ observed a simple Cotton effect for phenylcarbamate-substituted D-glucose, which indicates that the exciton splitting in ATC arises from the exciton interaction between phenyl carbamate groups attaching to neighboring glucose residues.

Since the local helix pitch of the amylosic chain $(=0.37 \mathrm{~nm})$ is shorter than that of the cellulosic chain $(=0.51 \mathrm{~nm})$, phenyl carbamate groups attaching to neighboring glucose residues are closer in the ATC chain, and the exciton interaction among the chromophores may be stronger than in the case of CTC. This may be the reason why the exciton peak of ATC persists up to higher temperature. The energetically favored helical states of cellulosic and amylosic chains are both left-handed, which may correspond to the same plus and minus signs of the CD signal at shorter and longer wavelengths for both ATC and CTC.

We measured CD spectra for fraction F18 $\left(M_{\mathrm{w}}=\right.$ $\left.5.87 \times 10^{4}\right)$ in THF at temperatures from 20 to -60 ${ }^{\circ} \mathrm{C}$, but observed no molecular weight dependence 
of CD. Furthermore, Bitteger and Keilich observed almost identical CD for ATC and maltose tris(phenyl carbamate). This indicates that the chiral arrangement of phenylcarbamate groups does not persist over so long range for both CTC and ATC.

Acknowledgment. We thank one reviewer to this article for his suggestion to describe clearly the difference between our work and Hsu et al.'s (ref 18). This work was partly supported by Grant-in-Aids for Scientific Research from the Japan Society for the Promotion of Science.

\section{REFERENCES}

1. M. M. Green, N. C. Peterson, T. Sato, A. Teramoto, R. Cook, and S. Lifson, Science, 268, 1860 (1995).

2. M. M. Green, J.-W. Park, T. Sato, A. Teramoto, S. Lifson, R. L. B. Selinger, and J. V. Selinger, Angew. Chem., Int. Ed., 38, 3138 (1999).

3. T. Nakano and Y. Okamoto, Chem. Rev., 101, 4013 (2001).

4. M. Fujiki, J. R. Koe, K. Terao, T. Sato, A. Teramoto, and J. Watanabe, Polym. J., 35, 297 (2003).

5. T. Sato, K. Terao, A. Teramoto, and M. Fujiki, Polymer, 44, 5477 (2003).

6. R. S. Werbowyj and D. G. Gray, Mol. Cryst. Liq. Cryst. (Lett.), 34, 97 (1976).

7. R. S. Werbowyj and D. G. Gray, Macromolecules, 13, 69 (1980).

8. Y. Okamoto and E. Yashima, Angew. Chem., Int. Ed., 37, 1020 (1998).

9. F. Kasabo, T. Kanematsu, T. Nakagawa, T. Sato, and A. Teramoto, Macromolecules, 33, 2748 (2000).

10. J. Danhelka, M. Netopilik, and M. Bohdanecky, J. Polym. Sci., Part B: Polym. Phys., 25, 1801 (1987).

11. T. Sato, T. Shimizu, F. Kasabo, and A. Teramoto, Macromolecules, 36, 2939 (2003).

12. T. Sato, M. Hamada, and A. Teramoto, Macromolecules, 36, 6840 (2003).

13. T. Kanematsu, T. Sato, Y. Imai, K. Ute, and T. Kitayama, Polym. J., 37, 65 (2005).

14. D. A. Brant and K. D. Goebel, Macromolecules, 8, 522 (1975).

15. K. D. Goebel, C. E. Harvie, and D. A. Brant, Appl. Polym. Symp., 28, 671 (1976).

16. D. A. Brant, Q. Rev. Biophys., 9, 527 (1976).

17. B. A. Burton and D. A. Brant, Biopolymers, 22, 1769 (1983).

18. B. Hsu, C. A. McWherter, D. A. Brant, and W. Burchard, Macromolecules, 15, 1350 (1982).

19. T. Sato, K. Terao, A. Teramoto, and M. Fujiki, Macromolecules, 35, 2141 (2002).

20. S. Lifson, C. Andreola, N. C. Peterson, and M. M. Green, J. Am. Chem. Soc., 111, 8850 (1989).

21. M. L. Mansfield, Macromolecules, 19, 854 (1986).

22. A. K. Gupta, E. Marchal, and W. Burchard, Macromolecules, 8, 843 (1975).

23. D. W. Tanner and G. C. Berry, J. Polym. Sci., Polym. Phys.
Ed., 12, 941 (1974).

24. J. W. Noordermeer, R. Daryanani, and H. Janeschitz-Kriegl, Polymer, 16, 359 (1975).

25. W. Burchard, Br. Polym. J., 3, 214 (1971).

26. S. Dayan, P. Maissa, M. J. Vellutini, and P. Sixou, Polymer, 23, 800 (1982).

27. H. Yamakawa and M. Fujii, Macromolecules, 7, 128 (1974).

28. H. Yamakawa and T. Yoshizaki, Macromolecules, 13, 633 (1980).

29. H. Yamakawa, "Helical Wormlike Chains in Polymer Solutions," Springer-Verlag, Berlin \& Heidelberg, 1997.

30. H. Yamakawa and W. H. Stockmayer, J. Chem. Phys., 57, 2843 (1972).

31. a) H. Yamakawa and J. Shimada, J. Chem. Phys., 83, 2607 (1985).

b) J. Shimada and H. Yamakawa, J. Chem. Phys., 85, 591 (1986)

32. T. Yoshizaki and H. Yamakawa, J. Chem. Phys., 81, 982 (1984).

33. S. S. C. Chu and G. A. Jeffrey, Acta Crystallogr., Sect. B: Struct. Sci., 24, 830 (1968).

34. C. V. Goebel, W. L. Dimpfl, and D. A. Brant, Macromolecules, 3, 644 (1970).

35. Y. Nakata, S. Kitamura, K. Takeo, and T. Norisuye, Polym. J., 26, 1085 (1994).

36. The torsional angles $\phi$ and $\psi$ in Figure 5a are defined as the dihedral angles formed by the atoms $\mathrm{H}(1) \mathrm{C}(1) \mathrm{O}(1) \mathrm{C}\left(4^{\prime}\right)$ and $\mathrm{C}(1) \mathrm{O}(1) \mathrm{C}\left(4^{\prime}\right) \mathrm{H}\left(4^{\prime}\right)$, respectively, measured relative to the cis conformation and being positive at the clockwise rotation along the vectors $\mathrm{C}(1) \mathrm{O}(1)$ and $\mathrm{O}(1) \mathrm{C}\left(4^{\prime}\right)$, respectively. In the three-bond model (in Figure $5 \mathrm{~b}$ ), the bond angles $\theta_{1}$, $\theta_{2}$, and $\theta_{3}(=\beta)$ are defined as the angles formed by bonds 1 and 2 , bonds 2 and 3 , and bonds 3 and 1, respectively, while the torsional angles $\tilde{\phi}_{1}$ and $\tilde{\phi}_{3}$ are defined as the dihedral angles formed by the three bonds $\mathbf{b}_{3} \mathbf{b}_{1} \mathbf{b}_{2}$ and $\mathbf{b}_{2} \mathbf{b}_{3} \mathbf{b}_{1}$, respectively, measured relative to the trans conformation and being positive at the clockwise rotation along the vectors $\mathbf{b}_{1}$ and $\mathbf{b}_{3}$, respectively. From the crystallographic data for cellobiose, ${ }^{33}$ we have the relations: $\tilde{\phi}_{1}=\psi+9.2^{\circ}$ and $\tilde{\phi}_{3}=\phi+6.6^{\circ}$.

37. D. A. Brant and W. L. Dimpfl, Macromolecules, 3, 655 (1970).

38. S. S. C. Chu and G. A. Jeffrey, Acta Crystallogr., 23, 1038 (1967).

39. As demonstrated by Burton and Brant, ${ }^{17}$ the configurational entropy $\Delta S_{\mathrm{c}}$ of the cellulosic chain is considerably larger than that of amylosic chain, but it should be noted that the $3 / 1$ and $2 / 1$ helical states in the cellulosic chain are not distinguished in $\Delta S_{\mathrm{c}}$ in contrast with the standard deviations $\left\langle\left(\tilde{\phi}_{i}-\tilde{\phi}_{i, 3 / 1}\right)^{2}\right\rangle^{1 / 2}$ and $\left\langle\left(\tilde{\phi}_{i}-\tilde{\phi}_{i, 2 / 1}\right)^{2}\right\rangle^{1 / 2}(i=2,3)$ in our argument.

40. R. C. Jordan, D. A. Brant, and A. Cesaro, Biopolymers, 17, 2617 (1978).

41. M. Fujii, K. Nagasaka, J. Shimada, and H. Yamakawa, Macromolecules, 16, 1613 (1983).

42. H. Bitteiger and G. Keilich, Biopolymers, 7, 539 (1969).

43. Y. Ashida, T. Sato, K. Morino, K. Maeda, Y. Okamoto, and E. Yashima, Macromolecules, 36, 3345 (2003). 